\title{
The half-twisted splice operation on reduced knot projections
}

\author{
Noboru Ito * Ayaka Shimizu ${ }^{\dagger}$
}

October 19, 2018

\begin{abstract}
We show that any nontrivial reduced knot projection can be obtained from a trefoil projection by a finite sequence of half-twisted splice operations and their inverses such that the result of each step in the sequence is reduced.
\end{abstract}

\section{Introduction}

Throughout this paper, knot projections are on $S^{2}$. A knot projection is a regular projection of a knot. Arnold [2], Calvo [4], and Endo-Itoh-Taniyama [6] introduced a local move that they, respectively, called perestroika, twisted splice, and smoothing operation, to describe a connection between plane curves or knot projections or diagrams. (See also [1], [3], [5], [7, [11, [12].) In this paper, we study a half-twisted splice on knot projections to create a connection between reduced knot projections.

A twisted splice on a knot projection is the replacement of trivial tangle with a two-arc braided tangle with $m$ crossings resulting in a knot projection as shown in Fig. 1, where $m$ is even (resp. odd) for segments oriented in the same (resp. opposite) direction [4]. We call the twisted splice with $m=1$,

\footnotetext{
*Department of Mathematics, Waseda University, 3-4-1 Okubo, Shinjuku-ku, Tokyo 169-8555, Japan. noboru@moegi.waseda.jp

${ }^{\dagger}$ Department of Mathematics, Hiroshima University, 1-3-1 Kagamiyama, HigashiHiroshima, 739-8526, Japan. shimizu1984@gmail.com
} 


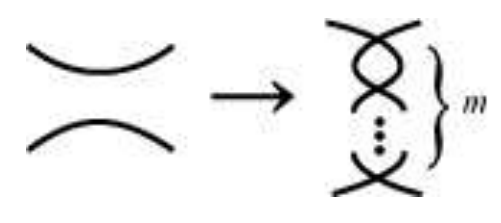

Figure 1: Twisted splice.

shown in Fig. 2, a half-twisted splice, and denote it by $A$. Obviously $A$ is a local move on knot projections. The inverse $A^{-1}$ is shown in the right-hand side of Fig. 2. Note that a twisted splice does not depend on the orienta-

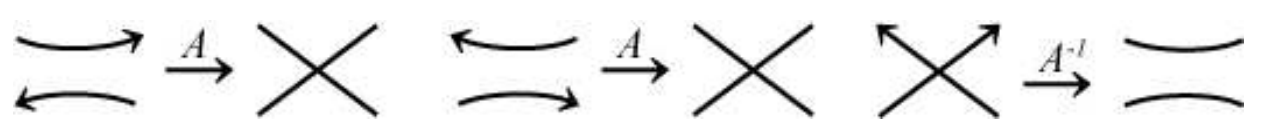

Figure 2: Half-twisted splice.

tion of knot projections, but depends only on the local "relative orientation". Hence $A$ and $A^{-1}$ also depend only on the relative orientation.

The trivial projection is the knot projection $O$, with no crossings, as depicted in the left-hand side of Fig. 3. The trefoil projection is the knot projection $P$ as depicted in the right-hand side of Fig. 3. We can obtain the trefoil projection by three half-twisted splices from the trivial projection as shown in Fig. 3. Every knot projection $P$ with $n$ crossings can be obtained

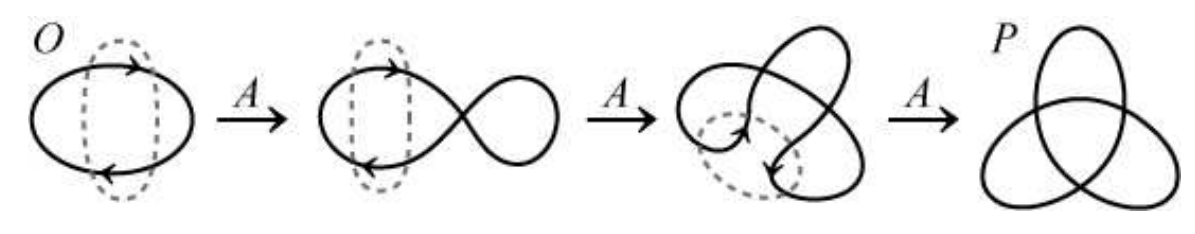

Figure 3: Half-twisted splices.

from a trivial projection by $n$ half-twisted splices because we obtain a knot projection with $(n-1)$ crossings from $P$ by a single $A^{-1}$. (This is suggested by Kawauchi, and see also [4].) 
A knot projection $P$ is reducible and has a reducible crossing $c$ if $P$ has a crossing point $c$ as shown in Fig. 4 , where $T$ and $T^{\prime}$ imply tangles. For example, the knot projections in the middle in Fig. 3 are reducible projections. A knot projection $P$ is reduced if $P$ is not reducible. Note that around a

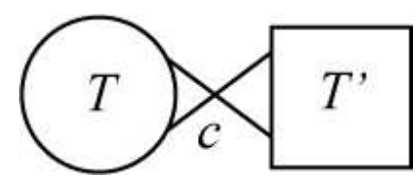

Figure 4: Reducible projection.

reducible (resp. non-reducible) crossing, there are exactly three (resp. four) disjoint regions.

In this paper, we shall prove the following theorem:

Theorem 1.1. Every nontrivial reduced knot projection is obtained from the trefoil projection by a finite sequence of $A$ and $A^{-1}$ such that the result of each step in the sequence is reduced.

This immediately gives us the following corollary:

Corollary 1.2. Let $P, Q$ be nontrivial reduced knot projections. There is a finite sequence of $A$ and $A^{-1}$ from $P$ to $Q$ such that the result of each step in the sequence is reduced.

This study was motivated by the knot game Region Select which was created by Kawauchi, Kishimoto and Shimizu. (This game is based on a local move, region crossing change [10.) In the game, we use knot projections. In particular, reduced knot projections make the game more interesting. A preliminary web-based version of Region Select in 2011 used $A$ to obtain a reduced knot projection from a reduced one. (The present version of the game can be found at http://www.sci.osaka-cu.ac.jp/math/OCAMI/news/gamehp/etop/gametop.html.) 
The rest of this paper is organized as follows: In Section 2, we introduce two new moves $B$ and $C$ and explore their relationship to $A$ and $A^{-1}$. In Section 3, we prove Theorem 1.1 by looking at the set of Reidemeister moves appropriate to oriented knot projections.

\section{The half-twisted splice and moves $B$ and $C$}

In this section, we discuss what we can accomplish with the operations $A$ and $A^{-1}$. We define the move $B$ to be the local move of a knot projection at

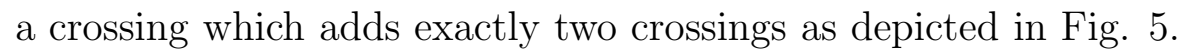

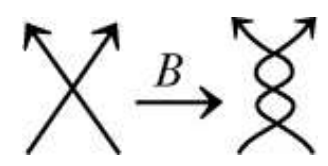

Figure 5: Definition of move $B$.

We have the following proposition:

Proposition 2.1. Let $P$ be a knot projection, and $c$ a crossing of $P$. Let $P^{\prime}$ be the knot projection obtained from $P$ by $B$ at $c$. We can obtain $P^{\prime}$ from $P$ by a finite sequence of $A$ and $A^{-1}$. Further, if $P$ is reduced, the result of each step in this sequence is also reduced.

Proof. See Fig. 6, If $P$ is reduced, then the regions $R_{1}, R_{2}, R_{3}$ and $R_{4}$ around $c$ are pairwise disjoint. Hence each step in the sequence of the local moves never create a reducible crossing.

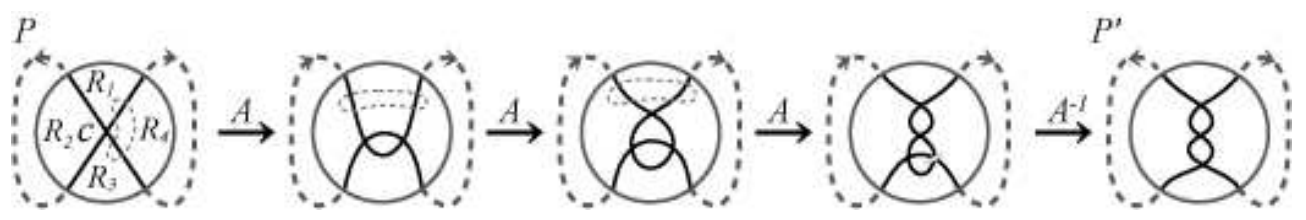

Figure 6: $B$ is realized by $A$ and $A^{-1}$. 
Next we consider connected sums. A connected sum $P \sharp Q$ of two knot projections $P$ and $Q$ is a knot projection as depicted in Fig. 7 . Note that the
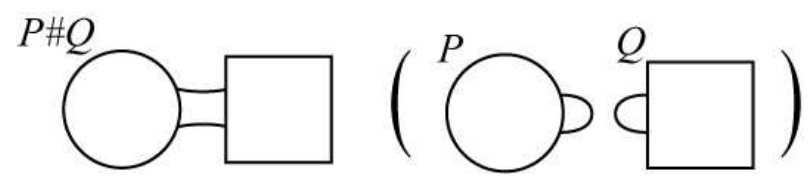

Figure 7: Connected sum.

connected sum is not unique. For example, the two connected sums in Fig. 8 are connected sums of a knot projection $P$ and a trefoil projection. The upper (resp. bottom) one is obtained by the connected sum at a triangle (resp. bigon) region of the trefoil projection. We have the following proposition:
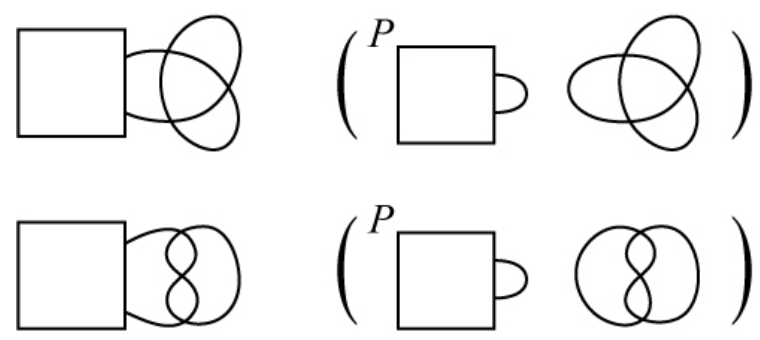

Figure 8: Connected sums with trefoil projection.

Proposition 2.2. The two types of connected sums with a trefoil projection are equivalent by a finite sequence of $A$ and $A^{-1}$ which create no reducible crossing.

Proof. See Fig. 9, Each crossing in Fig. 9 is non-reducible, and each box represents the same tangle.

Now we define moves $C$ and $C^{-1}$ to be the local moves shown in Fig. 10, Namely, $C$ is the connected sum with a trefoil projection, and $C^{-1}$ is the 


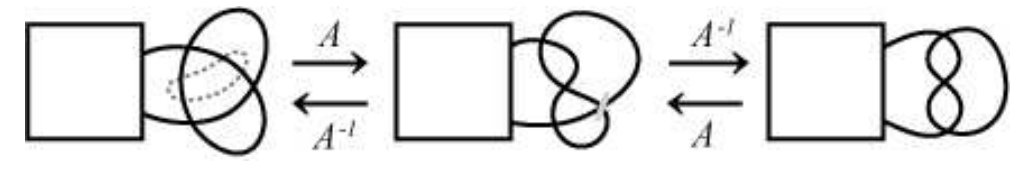

Figure 9: Connected sums with a trefoil projection.

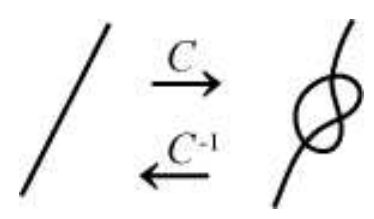

Figure 10: Definition of move $C$.

"removing" a trefoil projection.

We have the following proposition:

Proposition 2.3. We can create $C$ anywhere on a non-trivial knot projection by a finite sequence of $A$ and $A^{-1}$. Further, if $P$ is reduced, the result of each step in this sequence is also reduced.

Proof. See Fig. 11. If $P$ is reduced, then the regions $R_{1}, R_{2}, R_{3}$ and $R_{4}$ are pairwise disjoint, and therefore all the crossings shown in Fig. 11 are non-reducible.

By repeated application of Proposition 2.3, we have the following corollary:

Corollary 2.4. Any connected sum of $n$ trefoil projections $(n \geq 2)$ is obtained from a trefoil projection by a finite sequence of $A$ and $A^{-1}$ such that the result of each step in the sequence is reduced. 

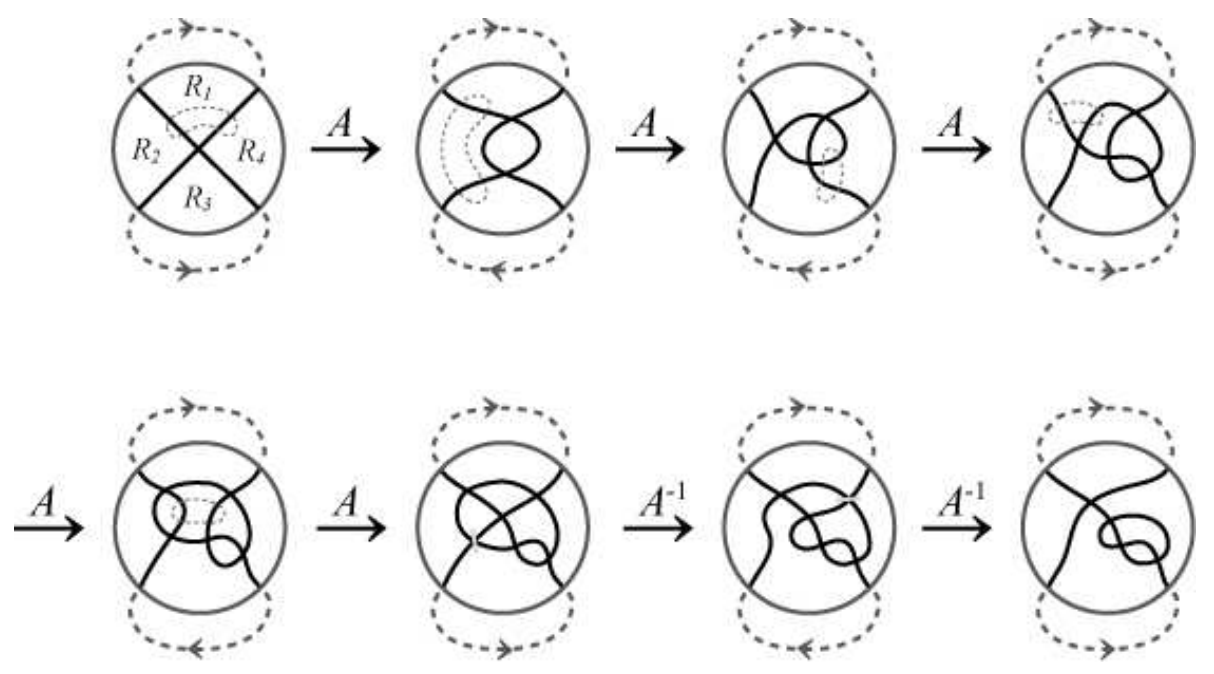

Figure 11: $C$ is realized by $A$ and $A^{-1}$.

\section{Proof of Theorem 1.1}

In this section, we prove Theorem 1.1. First, we consider Reidemeister moves on oriented knot diagrams and projections by refering to Polyak's theorem. Polyak [9] showed that, if $D$ and $D^{\prime}$ are diagrams in $\mathbb{R}^{2}$ representing the same oriented link, then $D^{\prime}$ is obtained from $D$ by a finite sequence of oriented Reidemeister moves $\Omega_{1 a}, \Omega_{1 c}, \Omega_{2 c}, \Omega_{2 d}$, and $\Omega_{3 b}$ as shown in Fig. 12.

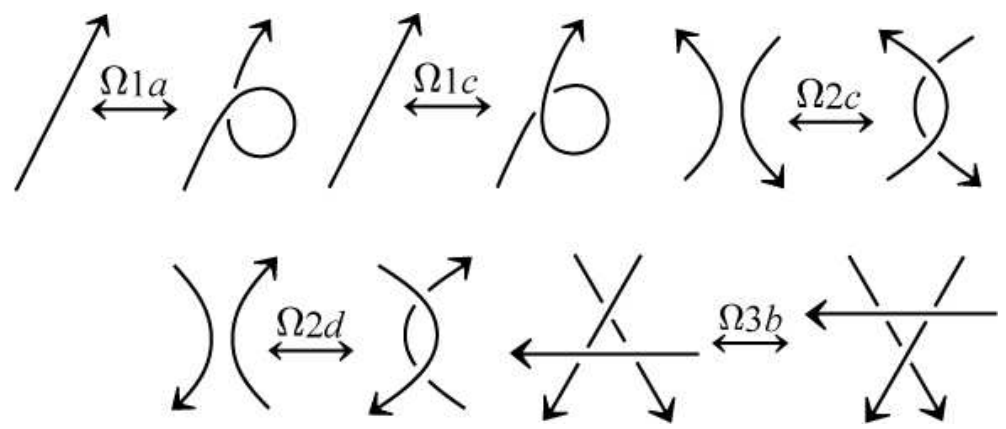

Figure 12: Reidemeister moves.

By erasing the over- and under-crossings in the diagram, we obtain ten dis- 
tinct Reidemeister moves on oriented projections. Remark that every knot projection can be made the projection of an unknot by an appropriate choice of crossings. In this setting, Polyak's theorem becomes:

Corollary 3.1. Any oriented knot projection can be obtained from the trivial projection by a finite sequence of the local moves $\Omega_{1+}, \Omega_{1-}, \Omega_{2+}, \Omega_{2-}, \Omega_{2^{\prime}+}$, $\Omega_{2^{\prime}-}, \Omega_{3+}$ and $\Omega_{3-}$ shown in Fig. 13.

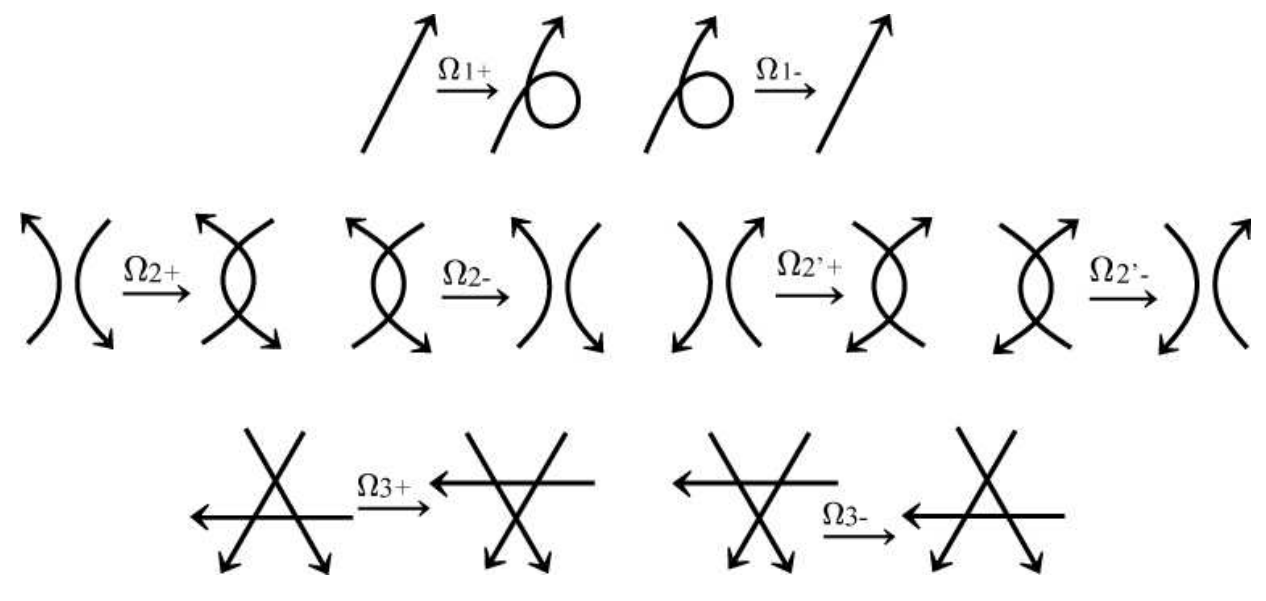

Figure 13: Reidemeister moves on projections.

Let $P$ be a knot projection. Let $P_{B}$ be the knot projection obtained from $P$ by applying $B$ at all the crossings of $P$. We have the following proposition:

Proposition 3.2. $P_{B}$ is a reduced knot projection even if $P$ is reducible.

Proof. Each reducible crossing of $P$ will be replaced with the three nonreducible crossings as shown in Fig. 14.

We have the following lemma: 


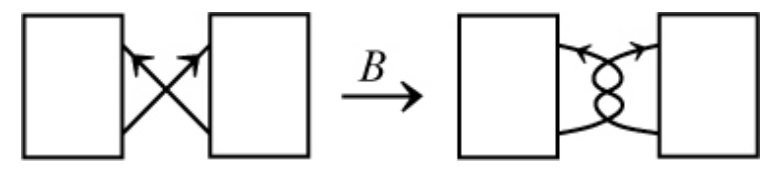

Figure 14: A reducible crossing will be replaced with non-reducible crossings.

Lemma 3.3. $P_{B}$ is obtained from the trefoil projection by a finite sequence of $A$ and $A^{-1}$ without ever passing through a reducible projection.

Proof. The moves $B$ and $C$ and their inverses are allowed in this proof since they each consist of a sequence of $A$ and $A^{-1}$. By Corollary 3.1, there exists a finite sequence of knot projections $P=P^{0} \rightarrow P^{1} \rightarrow P^{2} \rightarrow \cdots \rightarrow P^{m}=O$, where each $\rightarrow$ is one of $\Omega_{1+}, \Omega_{1-}, \Omega_{2+}, \Omega_{2-}, \Omega_{2^{\prime}+}, \Omega_{2^{\prime}-}, \Omega_{3+}$ and $\Omega_{3-}$. Remark that the last step of the sequence is $\Omega_{1-}, \Omega_{2-}$ or $\Omega_{2^{\prime}-}$. We can replace the $\Omega_{2-}$ move with a pair of $\Omega_{1-}$ moves if the last step is $\Omega_{2-}$ move. Similarly, we can replace the $\Omega_{2^{\prime}}$ - move with a pair of $\Omega_{1-}$ moves by reversing the orientation of the knot projection if the last step is $\Omega_{2^{\prime}}$ - move. Hence we can assume that $P^{m-1}$ is the knot projection with exactly one crossing. Let $P_{B}^{i}$ be the knot projection obtained from $P^{i}$ by applying $B$ at all the crossings of $P^{i}(i=0,1, \ldots, m)$. Note that $P_{B}^{m-1}$ is the trefoil projection. Now we show that we can move from $P_{B}^{i}$ to $P_{B}^{i+1}$ by a finite sequence of $A$ and $A^{-1}$ without ever passing through a reducible projection $(i=0,1, \ldots, m-1)$.

Case 1: Reidemeister move $\Omega_{1+}$. Use move $C$ on $P_{B}^{i}$ as in Fig. 15,
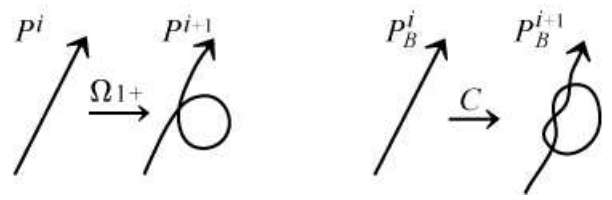

Figure 15: $\Omega_{1+}$ with $B$ is realized by $C$.

Case 2: Reidemeister move $\Omega_{1-}$. Use move $C^{-1}$ on $P_{B}^{i+1}$ as in Fig. 16. 


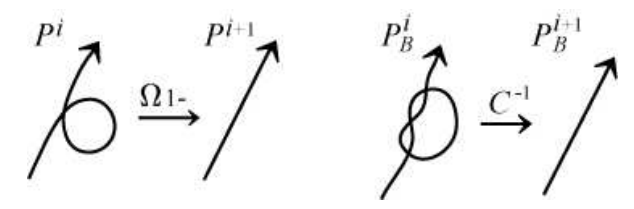

Figure 16: $\Omega_{1-}$ with $B$ is realized by $C^{-1}$.

Case 3: Reidemeister move $\Omega_{2+}$. See Fig. 17. Remark that $P_{B}^{i}$ is a nontrivial projection. Hence we can apply $C$ to the projection. Note that all the crossings shown in the figure are non-reducible crossings.

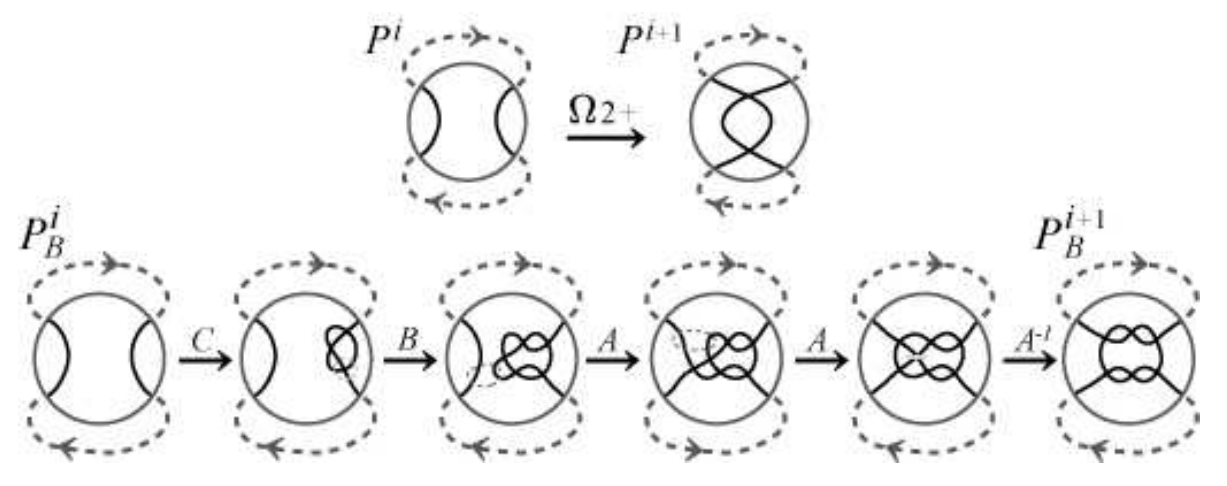

Figure 17: $\Omega_{2+}$ with $B$ is realized by $A$ and $A^{-1}$.

Case 4: Reidemeister move $\Omega_{2-}$. See Fig. 17 in the reverse order.

Case 5: Reidemeister move $\Omega_{2^{\prime}}$ Consider $\Omega_{2+}$ with the orientation of a projection reversed.

Case 6: Reidemeister move $\Omega_{2^{\prime}-}$. Similarly, consider $\Omega_{2-}$ with the orientation reversed.

Case 7: Reidemeister move $\Omega_{3+}$. See Fig. 18 and 19, Note that all the crossings shown in the figures are non-reducible crossings.

Case 8: Reidemeister move $\Omega_{3-}$. Similar to the case $\Omega_{3+}$. 


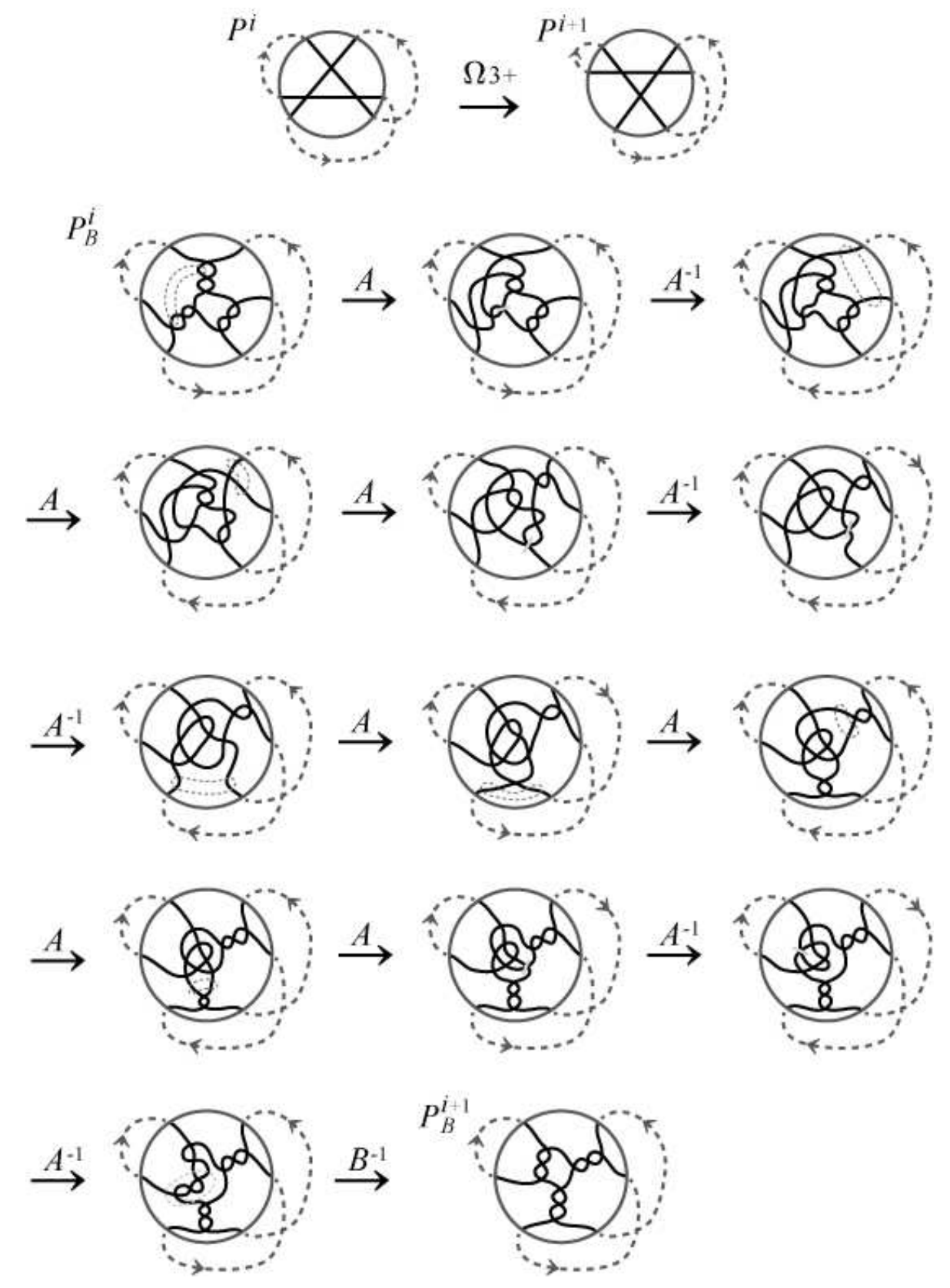

Figure 18: $\Omega_{3+}$ with $B$ is realized by $A$ and $A^{-1} \mathrm{I}$. 


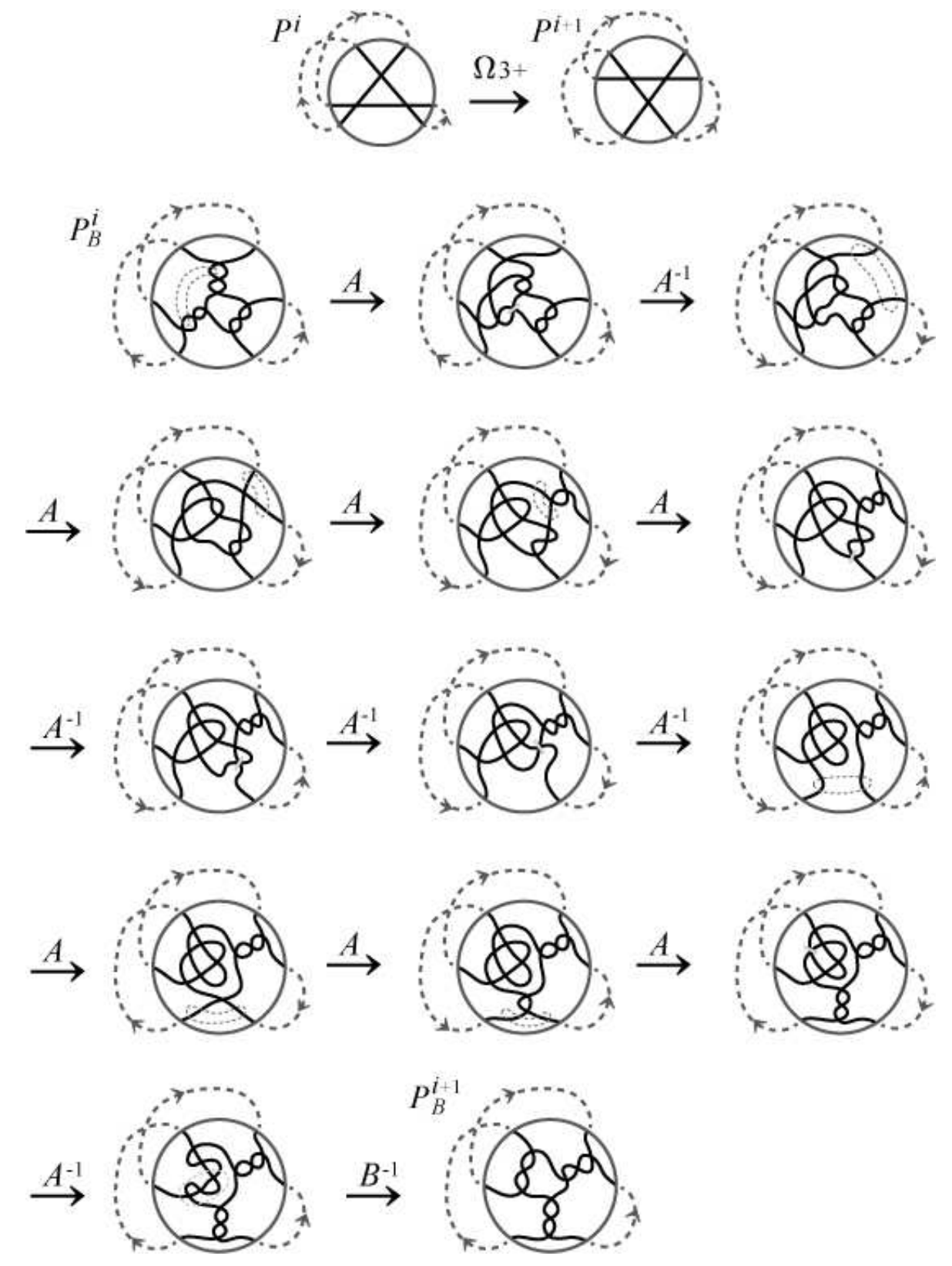

Figure 19: $\Omega_{3+}$ with $B$ is realized by $A$ and $A^{-1}$ II. 
We prove Theorem 1.1:

Proof of Theorem 1.1. We can transform $P$ into $P_{B}$ by Proposition 2.1, Then, we can transform $P_{B}$ by a finite sequence of $A$ and $A^{-1}$ into the trefoil projection by Lemma 3.3. In each case, the result of each step in the sequence will be a reduced projection.

\section{Acknowledgment}

N.I. was supported by Grant-in-Aid for Young Scientists (B) (23740062). A.S. thanks the members of Friday Seminar on Knot Theory 2011 at Osaka City University for valuable discussions and advice. In particular, she thanks Professor Akio Kawauchi for many helpful suggestions and giving her information about local moves on knot projections. She is deeply grateful to Professor Kenneth C. Millett for valuable discussions, advice, encouragement, and introducing her to Calvo's paper. She also thanks the referee for the kind advice and suggestions. She was partly supported by JSPS Research Fellowship for Young Scientists.

\section{References}

[1] E. Arnold, C. E. Fan, S. Pierre and C. C. Torres, Towards an Enumeration of Knots, unpublished research from the Summer Academic Research Internship at U. C. Santa Barbara (1990).

[2] V. I. Arnold, Plane curves, their invariants, perestroikas and classifications, Adv. Sov. Math. 21 (1994) 33-91.

[3] V. I. Arnold, Topological invariants of plane curves and caustics, University Lecture Series 5 (1994) AMS, Providence, RI.

[4] J. A. Calvo, Knot enumeration through flypes and twisted splices, $J$. Knot Theory Ramifications 6 (1997) 785-798. 
[5] J. A. Calvo, J. Bandrea, J. Golingo and S. Salton, Implementing an Enumeration of Prime Knots, unpublished research from the Summer Academic Research Internship at U. C. Santa Barbara (1991).

[6] T. Endo, T. Ito and K. Taniyama, A graph-theoretic approach to a partial order of knots and links, Topology Appl. 157 (2010) 1002-1010.

[7] J. Hoste, The enumeration and classification of knots and links, in Handbook of Knot Theory, eds. W. Menasco and M. Thistlethwaite (Elsevier, Amsterdam, 2005), pp. 209-232.

[8] A. Kawauchi, A survey of knot theory, Birkhauser, (1996).

[9] M. Polyak, Minimal generating sets of Reidemeister moves, Quantum Topology 1 (2010) 399-411.

[10] A. Shimizu, Region crossing change is an unknotting operation, arXiv:1011.6304.

[11] K. Taniyama, A partial order of knots, Tokyo J. Math. 12 (1989) 205229 .

[12] K. Taniyama, A partial order of links, Tokyo J. Math. 12 (1989) 475484. 